\title{
Anti-Saccades in Cerebellar Ataxias Reveal a Contribution of the Cerebellum in Executive Functions
}

\begin{abstract}
Elena Pretegiani ${ }^{1,2}$, Pietro Piu ${ }^{1}$, Francesca Rosini ${ }^{1,3}$, Pamela Federighi ${ }^{1,4}$, Valeria Serchi', Gemma Tumminelli ${ }^{1,3}$, Maria Teresa Dotti ${ }^{3}$, Antonio Federico ${ }^{3}$ and Alessandra Rufa ${ }^{1,3 *}$

'Eye-Tracking and Visual Application Laboratory (EVALab), Department of Medicine, Surgery and Neurosciences, University of Siena, Siena, Italy, ${ }^{2}$ Laboratory of Sensorimotor Research, National Eye Institute, National Institutes of Health, Department of Health and Human Services, Bethesda, MD, United States, ${ }^{3}$ Neurological and Neurometabolic Unit, Department of Medicine, Surgery and Neurosciences, University of Siena, Siena, Italy, ${ }^{4}$ Department of Business and Law, University of Siena, Siena, Italy
\end{abstract}

Objective: Increasing evidence suggests a cerebellar contribution to modulate cognitive aspects of motor behavior and executive functions. Supporting findings come from studies on patients with neurodegenerative diseases, in which however, given the extent of the disease, the specific role of the cerebellum, could not be clearly isolated. Anti-

OPEN ACCESS

Edited by: Caroline Tilikete,

Institut National de la Santé et de la Recherche Médicale (INSERM), France

Reviewed by: Delphine Lévy-Bencheton, The University of Queensland, Australia Jutta Peterburs,

Universität Münster, Germany

${ }^{*}$ Correspondence: Alessandra Rufa rufa@unisi.it

Specialty section:

This article was submitted to

Neuro-Ophthalmology,

a section of the journa

Frontiers in Neurology

Received: 22 November 2017 Accepted: 06 April 2018

Published: 23 April 2018

Citation:

Pretegiani E, Piu P, Rosini F, Federighi P, Serchi V, Tumminelli G, Dotti MT, Federico A and Rufa A (2018) Anti-Saccades in Cerebellar Ataxias Reveal a Contribution of the Cerebellum in Executive Functions.

Front. Neurol. 9:274.

doi: 10.3389/fneur.2018.00274 saccades are considered a sensitive tool to test executive functions. The anti-saccade underlying neural network, consisting of different cortical areas and their downstream connections including the lateral cerebellum, has been largely clarified. To separate the role of the cerebellum with respect to other cortical structures in executive control, we compared the anti-saccade performances in two distinct cohorts of patients with cerebellar disorders (with and without cerebral cortical involvement).

Methods: Eye movements during the execution of anti-saccades were recorded in 12 patients with spinocerebellar ataxia type 2 (a cortical-subcortical neurodegenerative disease), 10 patients with late onset cerebellar ataxia (an isolated cerebellar atrophy), and 34 matched controls.

Results: In the anti-saccade task, besides dynamic changes already demonstrated in the pro-saccades of these patients, we found in both groups of cerebellar patients prolonged latency with larger variability than normal and increased directional error rate. Errors, however, were corrected by cerebellar patients as frequently as normal. No significant differences were found in patients with and without cortical involvement.

Conclusion: Our results indicate, in a large cohort of cerebellar patients, that the cerebellum plays a critical role in the regulation of executive motor control not only, as well known, by controlling the end of a movement, but also modulating its initiation and reducing reflexive responses that would perturb voluntary actions.

Keywords: frontocerebellar network, goal-directed actions, cognitive functions, latency, error

Abbreviations: ACC, anterior cingulate cortex; BG, Basal Ganglia; DLPC, dorso-lateral pre-frontal cortex; FEF, frontal eye field; ICARS, International Cooperative Ataxia Rating Scale; LOCA, late-onset cerebellar ataxia; OMV, oculomotor vermis; PEF, parietal eye field; PPC, posterior parietal cortex; SCA2, spinocerebellar ataxia type 2; SEF, supplementary eye field; SMA, supplementary motor area; TMS, transcranial magnetic stimulation. 


\section{INTRODUCTION}

The cerebellum plays a well-known role in adapting motor responses by controlling the gain of a movement, its accuracy preservation, and by learning from endpoint errors (1-4). In the motor system, adaptive behavior is considered the result of cerebellar internal models. These models predict the sensory consequences of an action by integrating an efference copy of its motor command (forward model). A growing number of studies, however, have suggested that cerebellar internal models might act not only in the motor but also in the sensory, behavioral, and cognitive domains (5-11). These observations support the theory that the cerebellum plays a central role in monitoring executive brain performances $(12,13)$. Nevertheless, to which extent and for which functions this theory is valid, and which cerebellar areas are involved, remains largely unclear.

The anti-saccade task (14) is considered a sensitive tool to test executive functions and provides an effective way of studying higher-level motor control. It requires the cancelation of a reflexive saccade toward a suddenly appearing target (pro-saccade) and the execution of a self-placed movement toward a mirrored nonmarked position (anti-saccade). Moreover, if a direction error is made (unsuppressed pro-saccade toward the target), a corrective anti-saccade is demanded. The neural network underlying anti-saccades, also important in the generation of pro-saccades, has been partially clarified and consists of different areas in the medial and lateral frontal cortex, the parietal cortex, the basal ganglia, and the cerebellum (15-23). Frontal lobe dysfunctions have been associated with a global disruption of the anti-saccade planning, resulting in increased latency and error rate and inability to correct errors (15).

Similar abnormalities, however, have recently been reported also in cerebellar patients. A few studies, indeed, have found increased directional error rate and prolonged latencies of antisaccades in patients with hereditary cerebellar ataxia (24-27), focal cerebellar lesions (28), and acquired degenerative cerebellar diseases (29-31). Therefore, while the medial cerebellum, dorsal vermis, and fastigial nucleus [oculomotor vermis (OMV)], is traditionally considered the structure where saccade dynamic is regulated online (32), the cerebellar hemispheres and dentate nuclei (lateral cerebellum), conventionally not considered part of the oculomotor system, have been proposed to play a role in monitoring high-level saccadic behaviors, including the antisaccades $(24,32-37)$.

The control of executive function, however, remains largely considered a frontal cortex activity, with the cerebellum having only a possible ancillary contribution. Unfortunately, most studies on anti-saccades in neurodegenerative cerebellar ataxias enrolled patients with involvement of other cerebral structures beyond the cerebellum. These studies, thus, did not help to disambiguate the cortical vs cerebellar source of impaired anti-saccade performance, leaving unresolved the question of the exact role of the cerebellum in the regulation of voluntary movements. Patients with cerebellar stroke, on the other side, have often confined lesion, but the acute dynamic of the damage might lead to different dysfunction and compensatory mechanism than those resulting from slowly degenerative diseases.
Moreover, most anti-saccadic studies on cerebellar patients have reported just the directional error frequency and the antisaccadic latency, but not the frequency and latency of corrective saccades. Reduced frequency of correction has been recently related with cortical involvement (38) and could be used to better distinguish the cerebral vs cerebellar contribution.

Therefore, we aimed to segregate the cerebellar vs cerebral contribution to voluntary movement by comparing the antisaccade performances of two homogeneous cohorts of patients with different cerebellar diseases: late onset cerebellar ataxia (LOCA) and spinocerebellar ataxia type 2 (SCA2). LOCA is a "pure cerebellar syndrome," usually sporadic (39), in which the neurodegenerative process is confined to the cerebellum, while autosomal dominant SCA2 (MIM 183090) is caused by the expansion of CAG repeats within the ATXN2 gene (MIM 601517) and it is clinically characterized by a "cerebellar-plus syndrome" (40, 41 ), where cerebellar atrophy is associated with cerebral cortical atrophy and brainstem involvement (42).

To better clarify the cerebellar influence to executive functions, we considered not only the anti-saccadic directional error rate and latency, but also the frequency of correction of the directional errors and the intersaccadic interval between erroneous pro-saccades and corrective anti-saccades. The anti-saccadic parameters were compared with those of 34 controls and correlated with disability scores.

We hypothesized that if the cerebellum plays just an ancillary role in executive control, with respect to other cortical areas (i.e., the frontal lobes), performances should be worse in the patients with both cerebellar and cerebral involvement than just isolated cerebellar atrophy. Conversely, if the cerebellum plays a limiting step in executive function, performances should be similarly affected in cerebellar patients with and without cortical involvement.

We found impaired increased directional error rate (with normal corrective frequency) in all cerebellar patients without significant differences between those with and without cortical involvement. Our study, thus, demonstrates in a large cohort of cerebellar patients, a crucial contribution of the cerebellum in managing executive functions by suppression of reflexive movements. Moreover, since the latency of anti-saccades in SCA2 and LOCA patients was significantly longer and variable than in controls, but not significantly different between the two groups, we suggest that the cerebellum is also involved in monitoring voluntary motor performance by controlling the onset of saccades until the ambiguity on its planning is resolved.

\section{MATERIALS AND METHODS}

\section{Patients and Controls}

Clinical and magnetic resonance imaging (MRI) data of patients are resumed in Table 1. Twelve patients with genetically confirmed SCA2 (9 males, 3 females), mean age 38.7 years (range $25-53$ years) and mean age at onset 30.5 years (range $17-44$ years); and 10 patients with LOCA (5 males, 5 females), mean age 42.4 years (range $27-53$ years), and mean age at onset 36.9 years (range 20-52 years) were enrolled in the study. All patients underwent a complete neurological, neuro-ophthalmological, 
TABLE 1 | Clinical, genetic, and magnetic resonance imaging (MRI) findings of spinocerebellar ataxia type 2 (SCA2) and late onset cerebellar ataxia (LOCA) patients.

\begin{tabular}{|c|c|c|c|}
\hline Subject & CAG repeats & ICARS & MRI \\
\hline SCA2-1 & 43 & $26 / 100$ & Severe brainstem and cerebellar atrophy \\
\hline SCA2-2 & 38 & $36 / 100$ & $\begin{array}{l}\text { Mild cortical moderate to severe } \\
\text { brainstem and cerebellar atrophy }\end{array}$ \\
\hline SCA2-3 & 36 & $59 / 100$ & Severe brainstem and cerebellar atrophy \\
\hline SCA2-4 & 39 & $42 / 100$ & Severe brainstem and cerebellar atrophy \\
\hline SCA2-5 & 37 & $15 / 100$ & Moderate brainstem and cerebellar atrophy \\
\hline SCA2-6 & 40 & $30 / 100$ & $\begin{array}{l}\text { Moderate to severe brainstem } \\
\text { and cerebellar atrophy }\end{array}$ \\
\hline SCA2-7 & 42 & $56 / 100$ & $\begin{array}{l}\text { Mild cortical, moderate to severe } \\
\text { brainstem, and cerebellar atrophy }\end{array}$ \\
\hline SCA2-8 & 35 & $36 / 100$ & Moderate brainstem and cerebellar atrophy \\
\hline SCA2-9 & 36 & $35 / 100$ & Severe brainstem and cerebellar atrophy \\
\hline SCA2-10 & 38 & $37 / 100$ & Pons and cerebellar atrophy \\
\hline SCA2-11 & 40 & $23 / 100$ & Pons and cerebellar atrophy \\
\hline SCA2-12 & 41 & 48/100 & $\begin{array}{l}\text { Severe brainstem and cerebellar } \\
\text { atrophy. Mild cortical atrophy }\end{array}$ \\
\hline LOCA-1 & & $28 / 100$ & Global cerebellar atrophy \\
\hline LOCA-2 & & $40 / 100$ & Global cerebellar atrophy \\
\hline LOCA-3 & & $35 / 100$ & Global cerebellar atrophy \\
\hline LOCA-4 & & $25 / 100$ & Global cerebellar atrophy \\
\hline LOCA-5 & & $43 / 100$ & Global cerebellar atrophy \\
\hline LOCA-6 & & $27 / 100$ & Global cerebellar atrophy \\
\hline LOCA-7 & & $27 / 100$ & Global cerebellar atrophy \\
\hline LOCA-8 & & $34 / 100$ & Global cerebellar atrophy \\
\hline LOCA-9 & & $41 / 100$ & Global cerebellar atrophy \\
\hline LOCA-10 & & $38 / 100$ & Global cerebellar atrophy \\
\hline
\end{tabular}

The table shows the scores of the International Cooperative Ataxia Rating Scale (ICARS), and the MRI outcomes for the LOCA and SCA2 patients. ICARS is a specifically validated scale, in the interval from 0 to 100 , to evaluate cerebellar ataxia, with higher values denoting major clinical anomalies. For the SCA2 patients, the number of trinucleotide repeats (CAG) is also reported.

and International Cooperative Ataxia Rating Scale (ICARS) (43) examination. All patients also underwent a structural brain MRI, evaluated separately by two experienced neuroradiologists, and the recommended clinical protocol for ataxias, including pertinent genetic and laboratory testing. In patients with LOCA, known causes of acquired and inherited ataxias were ruled out (Friedreich ataxia, fragile X-premutation, ataxia-telangiectasia, ataxias associated with mutation of aprataxin, senataxin or sacsin, metabolic causes of ataxia such as abetalipoproteinemia, vitamin E deficiency, late-onset Tay-Sachs disease, cerebrotendinous xanthomatosis, autosomal dominant spinocerebellar ataxias due to de novo mutations). Only two SCA2 patients (old relatives of two patients included in the study) with qualitatively severe cortical atrophy and cognitively compromised (MMSE <23) were excluded from the study. The control group consisted of 34 healthy age-matched subjects ( 13 males, 21 females) of mean age 36 years (range 19-65 years). All subjects gave their informed consent. The study respected the Declaration of Helsinki and was approved by the local Ethics Committee.

\section{Eye Movement Recording}

Eye movements were recorded with an ASL 504 eye-tracker device (Applied Science Laboratories, Bedford, MA, USA). Data acquisition and visual stimulation were controlled by a PC $(3 \mathrm{GHz}$ Pentium) running a custom software dedicated to real-time data acquisition. Eye position was sampled at $240 \mathrm{~Hz}$, digitized with a resolution of 16 bits, corresponding to a sensitivity of recorded eye position of $0.16 \mathrm{deg}$, and stored for off-line analysis. The visual stimulus was a red dot (luminance $63 \mathrm{~cd} / \mathrm{m}^{2}$ ) with a diameter subtending a visual angle of $0.4 \mathrm{deg}$, presented on a black background (luminance $2.5 \mathrm{~cd} / \mathrm{m}^{2}$ ). We used a $310 \times 510 \mathrm{~mm}$ LCD screen, with a resolution of $1,024 \times 768$ pixels, positioned $720 \mathrm{~mm}$ from the subject's eyes.

The subjects were seated in a darkened room while their head movements were minimized by a chinrest with a bite bar. Each recording session was preceded by an interactive calibration procedure based on nine static points and three static points of validation.

\section{Anti-Saccade Task}

Each anti-saccade task consisted in a block of 40 trials per session, with a total of 80 trials. During each trial, a central fixation point was presented for $500 \mathrm{~ms}$. After the disappearance of the central fixation point, a peripheral target was presented randomly with respect to direction (left, right) and position (10, 18 degrees) for 2,500 ms. We chose two different target eccentricities (10 and 18 degrees) since possible different neural networks might control smaller and larger saccades (44).

Subjects were instructed to make a mirror saccade to the opposite direction of the target. To reduce memory engagement during the task, the correct anti-saccade position was unmarked after the anti-saccade landing, and it was followed by the appearance of the central fixation point. At this moment, the subject was instructed to return to the center of the screen. The trials were obtained in two different sessions (same day or the day after) depending on the patient's compliance. All trials were analyzed by two expert neurologists (Francesca Rosini and Gemma Tumminelli).

\section{Signal Processing}

Signal processing was conducted off-line using a semi-automatic detection algorithm. Data were filtered using a third-order Butterworth low-pass digital filter with $-3 \mathrm{~dB}$ attenuation at $25 \mathrm{~Hz}$ cut-off frequency. A velocity threshold of $10 \mathrm{deg} / \mathrm{s}$ was used to determine the starting and ending times of saccades. Eye velocity was obtained with an eight-point central difference derivative algorithm having a bandwidth larger than $70 \mathrm{~Hz}$ at a digitization frequency of $240 \mathrm{~Hz}$ (45). Saccades with latency less than $100 \mathrm{~ms}$, were considered anticipatory and excluded. We excluded all oblique saccades whose direction had an angle $>30^{\circ}$ with respect to the reference of the horizontal plane. In the analyzed groups, the number of saccades that did not meet one or more of these criteria was no more than the $15 \%$ of all saccades performed by each subject. The saccades were automatically identified through an ad hoc software and, subsequently, manually validated by two trained investigators. Saccades detection and parameter estimate algorithms were performed using Matlab software, version 7 (The MathWorks Inc., Natick, MA, USA).

\section{Saccade Definitions, Detection, and Analysis}

In the anti-saccade task, the subjects are asked to execute an eye movement in the opposite direction from a visual stimulus onset. 
This movement implies the suppression of the reflexive tendency to look at the sudden onset stimulus (pro-saccade). Accordingly, we can give the following definitions: (a) a correct anti-saccade is the saccadic eye movement directed to the opposite direction in respect to visual target; (b) an erroneous pro-saccade is an uninhibited reflexive saccade toward the target; and (c) a corrective anti-saccade is an anti-saccade made after an initial erroneous pro-saccade.

For the analysis of the saccadic movements, we considered the following set of parameters: duration, peak and mean velocity, gain, and latency. The duration was calculated as the time interval between the start and the end of the movement; the peak velocity was the maximum eye velocity, in degrees of visual angle/second; gain was the ratio of the initial saccade amplitude to target distance, where we defined saccadic amplitude as the difference, in degrees of visual angle, between the eye position at the start and end of the saccade. The saccade latency was defined as the time delay between the target presentation and the saccade onset, and it was calculated also for erroneous pro-saccades and corrective anti-saccades. The latency of corrective anti-saccades includes the latency of an erroneous pro-saccade, its duration and the latency of the subsequent corrective anti-saccade. Moreover, we determined the intersaccadic latency between the end of the erroneous pro-saccade and the start of the corrective anti-saccade, when executed. Both measures (latency of corrective anti-saccades and intersaccadic latency) reflect anti-saccade programming. However, the intersaccadic latency is a more precise estimation of the anti-saccade programming. Indeed, if it is longer than the visual feedback of the error, the corrective anti-saccade is likely a movement generated after the visual error; if the intersaccadic latency is shorter than the visual feedback or the corresponding average latency of a correct anti-saccade, it is likely that the corrective anti-saccade is a pre-programmed movement generated before the visual feedback of the error.

Means and SDs of all saccadic parameters mentioned above were calculated for each task of each patient.

With respect to the gain, the mean value indicates the accuracy of the anti-saccades (closeness of the average amplitude to the actual target), while the reciprocal of the SD indicates the precision of the anti-saccades (compactness of grouping, or consistency, among amplitudes). Precisely, the greater the SD, the lower is the precision.

\section{Statistical Methods}

Spearman rank order correlation coefficients were computed to measure the association among patient's disability score (ICARS scale) and saccadic parameters. Either Kruskal-Wallis tests or Welch tests on ranked data were used to compare the effect of the group membership (controls, SCA2, and LOCA) on each saccadic parameter at 10 and 18 degrees and their variability among groups. Levene tests were used to assess homoscedasticity. A post hoc analysis was performed by Dunn multiple comparison test with Bonferroni adjustment. To verify the null hypothesis that the latencies of correctly executed anti-saccades were sampled from the same continuous distribution, Kolmogorov-Smirnov tests were set for pairwise comparisons of the latency distributions (controls vs LOCA, controls vs SCA2, LOCA vs SCA2).
The amplitude-duration and amplitude-peak velocity main sequences of the anti-saccades were considered. An exponential equation was used to fit peak velocity on amplitude, i.e., $V_{\text {peak }}=V_{\max } *\left[1-e^{\text {Amplitude } / c}\right]$, where $V_{\max }$ is the asymptotic peak velocity and $c$ is a constant. A linear fitting, i.e., Duration $=k+b *$ Amplitude, where $k$ is a constant and $b$ is the slope of the fitted line, was adopted for the amplitude-duration relationship. The $95 \%$ prediction bounds around the healthy controls' main sequences were also calculated. Percentage of direction errors with respect to the total number of anti-saccades trials and percentage of corrective anti-saccades with respect to the number of direction errors were compared among the three groups through $\chi^{2}$ tests (with two degrees of freedom). The Marascuilo procedure was applied to identify which proportions could be responsible for rejecting the null hypothesis of equal ratios. All tests were two-sided at the nominal significance level of $5 \%$.

\section{RESULTS}

\section{Clinical and MRI Data}

ICARS scores, MRI findings, and number of CAG repeats (for SCA2) of all patients are shown in Table 1. All SCA2 patients had been previously diagnosed by molecular analysis demonstrating abnormal CAG expansion (46). In these patients, MRI examination showed cerebellar atrophy extending to the middle cerebellar peduncle and midbrain, and the dorsal pons. Mild diffuse cortical atrophy was qualitatively observed in all SCA2 patients by two experienced neuroradiologists. In our SCA2 patients, while abnormality of the cerebral cortex was only qualitatively evaluated, but not quantitatively measured at the brain MRI, a cortical functional impairment could not be excluded given the common involvement in this disease. No basal ganglia changes were observed in these patients. No frank cognitive disabilities or dementia (MMSE score was $\geq 24$ in all patients) were detected. Mean ICARS score in SCA2 population was 36.9/100. All LOCA subjects presented with pure cerebellar syndrome and MRI evidence of global cerebellar atrophy; no extra-cerebellar involvement was evident at MRI. Mean ICARS score in LOCA population was 33.8/100. No significant association between clinical disability assessment and anti-saccadic parameters were found in SCA2 and LOCA.

\section{Anti-Saccades}

To better represent the results of anti-saccadic behavior, we are reporting separately the dynamic and metric outcome of the analysis, the latency, and the different measures of accuracy (Tables 2-4).

\section{Dynamic Parameters}

Late onset cerebellar ataxia patients showed slower anti-saccades than LOCA and controls (Table 2). In details: no differences were observed between controls and LOCA in average velocity and duration. SCA 2 patients showed: (a) longer durations than controls (10 deg, $p<0.001 ; 18 \mathrm{deg}, p<0.001$ ) and LOCA patients (18 deg, $p<0.001$ ); (b) lower peak velocities than controls and LOCA (10 deg, $p<0.001 ; 18 \mathrm{deg}, p<0.001$ ); and (c) lower 
mean velocity than controls and LOCA (10 deg, $p<0.001$; $18 \mathrm{deg}, p<0.001$ ) (Table 2). The SD of the anti-saccade dynamic parameters of healthy controls resulted lower than SCA2 (10 deg, $p<0.001 ; 18 \mathrm{deg}, p<0.001)$ and LOCA (10 deg, $p=0.009)$ (Table 3). The anti-saccades followed the main sequence relationships (peak velocity vs amplitude and duration vs amplitude, Figures 1A,B) in LOCA and control groups with no significant differences; SCA2 patients, indeed, did not follow the main sequence. In particular, SCA2 patients showed significantly lower peak velocity $\left(V_{\max }=496.0 \mathrm{deg} / \mathrm{s} \pm 73.3 \mathrm{deg} / \mathrm{s}, C=34.6 \pm 7.2\right)$ compared with healthy controls $\left(V_{\max }=424.4 \mathrm{deg} / \mathrm{s} \pm 8.2 \mathrm{deg} /\right.$ $\mathrm{s}, C=7.0 \pm 0.5)$ and LOCA $\left(V_{\max }=551.4 \mathrm{deg} / \mathrm{s} \pm 18.4 \mathrm{deg} / \mathrm{s}\right.$, $C=11.4 \pm 0.8)$. In the amplitude-duration main sequence the duration in SCA2 patients $(k=104.8 \mathrm{~ms} \pm 7.2 \mathrm{~ms}, b=3.7 \pm 0.3)$ resulted greater than LOCA $(k=41.1 \mathrm{~ms} \pm 2.6 \mathrm{~ms}, b=2.0 \pm 0.1)$ and controls $(k=22.5 \mathrm{~ms} \pm 2.5 \mathrm{~ms}, b=3.4 \pm 0.1)$.

\section{Accuracy and Precision}

The cerebellar patients showed overall good accuracy of antisaccades, but worse precision than normal. Accuracy of correctly executed anti-saccades did not differ significantly among

TABLE 2 | Mean and SD values of dynamic and metric anti-saccade parameters.

\begin{tabular}{lccccc}
\hline & & $\begin{array}{c}\text { Duration } \\
(\mathbf{m s})\end{array}$ & $\begin{array}{c}\text { Peak velocity } \\
\text { (deg/s) }\end{array}$ & $\begin{array}{c}\text { Mean } \\
\text { velocity } \\
\text { (deg/s) }\end{array}$ & Gain [-] \\
\hline CTR & $10 \mathrm{deg}$ & $64.2 \pm 19.3$ & $339.2 \pm 63.7$ & $179.2 \pm 35.7$ & $0.98 \pm 0.31$ \\
& $18 \mathrm{deg}$ & $82.2 \pm 11.9$ & $433.3 \pm 72.3$ & $234.1 \pm 38.0$ & $0.96 \pm 1.14$ \\
\hline SCA2 & $10 \mathrm{deg}$ & $132.6 \pm 58.9$ & $202.2 \pm 61.7$ & $107.6 \pm 30.4$ & $1.08 \pm 0.41$ \\
& $18 \mathrm{deg}$ & $178.7 \pm 40.4$ & $191.6 \pm 91.2$ & $99.9 \pm 45.1$ & $0.83 \pm 0.27$ \\
\hline LOCA & $10 \mathrm{deg}$ & $69.4 \pm 12.1$ & $379.6 \pm 64.3$ & $199.7 \pm 33.6$ & $1.22 \pm 0.41$ \\
& $18 \mathrm{deg}$ & $85.9 \pm 19.4$ & $471.2 \pm 98.1$ & $250.0 \pm 53.9$ & $0.94 \pm 0.34$
\end{tabular}

Mean and SDs of duration, peak velocity, mean velocity, and gain of correctly executed anti-saccades estimated for each group (CTR, control group) separately at 10 and 18 deg. Statistical significance of comparisons among groups.

Duration: spinocerebellar ataxia type 2 (SCA2) vs CTR (10 and $18 \mathrm{deg}, p<0.001$ ); SCA2 vs late onset cerebellar ataxia (LOCA) (18 deg, $p<0.001)$.

Peak velocity: SCA2 vs CTR (10 and 18 deg, $p<0.001$ ); SCA2 vs LOCA (10 and 18 deg, $p<0.001$ ).

Mean velocity: SCA2 vs CTR (10 and $18 \mathrm{deg}, p<0.001)$; SCA2 vs LOCA (10 and 18 deg, $p<0.001$ ). cerebellar patients (Table 2). Controls revealed significant higher precision in gain than LOCA (10 deg, $p=0.001)$ and SCA2 (10 deg, $p<0.001 ; 18 \mathrm{deg}, p=0.007)$ (Table 3).

\section{Latency}

The cerebellar patients showed increased latency of all saccades (correct and corrective anti-saccades and pro-saccades) and longer intersaccadic intervals between erroneous pro-saccade and corrective anti-saccades (Table 4). The average latency of correctly executed anti-saccades was longer in SCA2 (10 deg, $p=0.003 ; 18 \mathrm{deg}, p<0.001)$ and LOCA (10 deg, $p=0.0012$; $18 \mathrm{deg}, p<0.001)$ than controls. The latency of erroneous pro-saccades did not differ among groups at $10 \mathrm{deg}$. Latency of erroneous pro-saccade was significantly longer in SCA2 than controls at $18 \mathrm{deg}(p=0.015)$. The latency of corrective antisaccades (with respect to the target onset) was significantly lower in the controls than LOCA (10 deg, $p=0.003 ; 18 \mathrm{deg}, p=0.005$ ) and SCA2 patients (10 deg, $p<0.001 ; 18 \mathrm{deg}, p=0.003$ ); no significant differences were observed between the two groups of patients. The intersaccadic latency was shorter in the controls than LOCA (10 deg, $p=0.004 ; 18 \mathrm{deg}, p=0.001$ ) and SCA2 (10 deg, $p=0.011 ; 18 \mathrm{deg}, p=0.002$ ), while no differences were observed between the two groups of patients (Table 4; Figures 2A-D).

The distribution of latency of correctly executed anti-saccades was significantly less broadened in controls than both LOCA $(p<0.001)$ and SCA2 $(p<0.001)$. The distribution of latency did not differ significantly between LOCA and SCA2 (Figure 3).

\section{Directional Error and Correction}

The cerebellar patients made more directional error than normal, but corrected as frequently as controls. Indeed, the direction error rate (Figure 4A) was found significantly different among groups (10 deg, $p<0.001 ; 18 \mathrm{deg}, p<0.001$ ). More precisely: (a) the error rate was higher in SCA2 (39.6\%) than controls (19.3\%) (percentage difference, 20.3; critical value, 10.61); (b) in LOCA (65.4\%) than controls (percentage difference, 46.1; critical value, 10.36); and (c) the percentage of errors in LOCA was significantly higher than in SCA2 (percentage difference, 25.82; critical value, 13.02). The percentage of corrections among healthy controls (10 deg, 97.2\%; $18 \mathrm{deg}, 100 \%)$, SCA2 (10 deg, 100\%; $18 \mathrm{deg}, 100 \%)$, and

TABLE 3 | SDs of anti-saccade parameters.

\begin{tabular}{|c|c|c|c|c|c|c|}
\hline & & Duration (ms) & Peak velocity (deg/s) & Mean velocity (deg/s) & Gain [-] & Latency (ms) \\
\hline \multirow[t]{2}{*}{ CTR } & 10 deg & $58.6(17.5)$ & $70.1(53.5)$ & $41.4(23.4)$ & $0.20(0.11)$ & $41.6(14.9)$ \\
\hline & $18 \mathrm{deg}$ & $80.7(17.4)$ & 78.9 (36.6) & $48.7(21.5)$ & $0.14(0.08)$ & $31.6(14.7)$ \\
\hline \multirow[t]{2}{*}{ SCA2 } & 10 deg & $117.8(64.6)$ & $53.3(9.3)$ & $24.6(5.9)$ & $0.47(0.08)$ & $35.4(58.1)$ \\
\hline & $18 \mathrm{deg}$ & $185.6(42.7)$ & $39.6(26.2)$ & $18.1(16.4)$ & $0.20(0.06)$ & $64.8(49.6)$ \\
\hline \multirow[t]{2}{*}{ LOCA } & 10 deg & $68.5(18.4)$ & $106.4(41.2)$ & $51.7(18.1)$ & $0.57(0.26)$ & $71.3(56.8)$ \\
\hline & $18 \mathrm{deg}$ & $83.3(23.0)$ & $93.5(43.2)$ & $56.0(22.0)$ & $0.18(0.11)$ & $62.8(60.9)$ \\
\hline
\end{tabular}

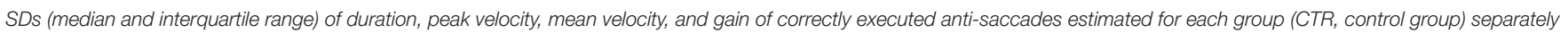
at 10 and 18 deg. Statistical significance of comparisons among groups.

Latency: spinocerebellar ataxia type 2 (SCA2) vs CTR (18 deg, $p<0.05)$.

Duration: SCA2 vs CTR (10 and $18 \mathrm{deg}, p<0.001)$; SCA2 vs late onset cerebellar ataxia (LOCA) (10 deg, $p<0.05 ; 18$ deg, $p<0.001)$.

Peak velocity: SCA2 vs CTR (18 deg, $p<0.001)$; LOCA vs controls (10 deg, $p<0.001$ ); SCA2 vs LOCA (10 deg, $p<0.05 ; 18$ deg, $p<0.001$ ).

Mean velocity: SCA2 vs LOCA (10 and $18 \mathrm{deg}, p<0.001)$; SCA2 vs controls (10 deg, $p<0.05 ; 18 \mathrm{deg}, p<0.001)$.

Gain: SCA2 vs CTR (10 deg, $p=0.001 ; 18$ deg, $p<0.05$ ); LOCA vs controls (10 deg, $p<0.001$ ). 
LOCA (10 deg, 98\%; 18 deg, 97.4\%) did not result in significant differences at both target eccentricities (Figure 4B).

\section{DISCUSSION}

The observation of executive functions impairment in cerebellar patients is one evidence in favor of a cerebellar role in cognitive activity $(25-27,30-32,47,48)$. Nevertheless, the role of the cerebellum in executive functions control has been poorly differentiated from that of cerebral cortical areas, in particular the frontal cortex.

Therefore, in this study, in order to better understand the role of the cerebellum in controlling the anti-saccades and ultimately its role in controlling executive functions, we compared the anti-saccadic behavior of two pathologically different groups of cerebellar neurodegenerative diseases: SCA2 with and LOCA without involvement of the frontal cortex. We found increased latency of anti-saccades in both groups of cerebellar patients

TABLE 4 | Mean and SD of latencies of correct anti-saccades, erroneous prosaccades, corrective anti-saccades, and intersaccadic intervals.

\begin{tabular}{lllccc}
\hline & & $\begin{array}{c}\text { Anti-saccade } \\
\text { latency }(\mathbf{m s})\end{array}$ & $\begin{array}{c}\text { Erroneous } \\
\text { pro-saccade } \\
\text { latency }(\mathbf{m s})\end{array}$ & $\begin{array}{c}\text { Corrective } \\
\text { anti-saccade } \\
\text { latency }(\mathbf{m s})\end{array}$ & $\begin{array}{c}\text { Intersaccadic } \\
\text { latency }(\mathbf{m s})\end{array}$ \\
\hline \multirow{2}{*}{ lTR } & $10 \mathrm{deg}$ & $268.7 \pm 30.8$ & $184.2 \pm 23.3$ & $363.4 \pm 43.6$ & $127.5 \pm 44.5$ \\
& $18 \mathrm{deg}$ & $267.3 \pm 28.3$ & $204.9 \pm 39.8$ & $370.5 \pm 67.1$ & $93.2 \pm 45.2$ \\
\hline \multirow{2}{*}{ SCA2 } & $10 \mathrm{deg}$ & $376.3 \pm 83.8$ & $260.4 \pm 88.9$ & $530.3 \pm 53.1$ & $252.6 \pm 87.3$ \\
& $18 \mathrm{deg}$ & $412.3 \pm 133.8$ & $264.3 \pm 49.1$ & $518.4 \pm 100.1$ & $219.4 \pm 46.7$ \\
\hline \multirow{2}{*}{ LOCA } & $10 \mathrm{deg}$ & $396.3 \pm 109.3$ & $213.8 \pm 35.6$ & $530.4 \pm 115.4$ & $240.4 \pm 117.7$ \\
& $18 \mathrm{deg}$ & $393.1 \pm 61.34$ & $236.9 \pm 29.9$ & $519.0 \pm 121.1$ & $223.4 \pm 77.9$
\end{tabular}

Mean and SDs of latencies of correctly executed anti-saccades, erroneous prosaccades, corrective anti-saccades, and intersaccadic intervals estimated for each group (CTR, control group) separately at 10 and $18 \mathrm{deg}$. Statistical significance of comparisons among groups.

Anti-saccades: spinocerebellar ataxia type 2 (SCA2) vs CTR (10 deg, $p<0.05 ; 18$ deg $p<0.001$ ); late onset cerebellar ataxia (LOCA) vs controls (10 and $18 \mathrm{deg}, p<0.001$ ). Erroneous pro-saccades: SCA2 vs CTR (18 deg, $p<0.05)$.

Corrective anti-saccades: SCA2 vs CTR (10 deg, $p<0.001 ; 18$ deg, $p<0.05)$; LOCA vs controls (10 and $18 \mathrm{deg}, p<0.05)$.

Intersaccadic: SCA2 vs CTR (10 and $18 \mathrm{deg}, p<0.05)$; LOCA vs controls (10 deg, $p<0.05 ; 18$ deg, $p=0.001$ ) with respect to controls. The cerebellar patients made also more directional errors, but their errors were corrected as frequently as controls. No significant differences were found in patients with and without cortical involvement. Other saccadic dynamic parameters of anti-saccades retained the same characteristics of pro-saccades [see Federighi et al. (49) for a description of prosaccades in these patients]. Indeed, according to impairment of the brainstem burst generator and OMV, SCA2 showed slower anti-saccades, and LOCA faster anti-saccades (50). Furthermore, metric parameters, such as velocity, were affected by a greater variability in both cerebellar patients than controls.

The anti-saccades are an effective tool to study executive functions, since their underlying neural network has been relatively well clarified. This often allows the specific identification of which step, and therefore related cerebral area, might be impaired in case of defective performance.

In particular, the frontal eye field (FEF) is supposed to plan the correct anti-saccade while, along with the dorso-lateral prefrontal cortex (51-53), it evaluates the appropriateness of the saccade response with respect to the specific context, avoiding inappropriate responses (54-59). The pre-supplementary motor area (pre-SMA) and supplementary eye field (SEF) (60-63) predispose the oculomotor system for the anti-saccade response over the prevalent pro-saccade reaction. The anterior cingulate cortex is also supposed to contribute to the anti-saccade planning by monitoring the resolution of conflict between reflexive and anti-saccades $(55,64)$. In the posterior parietal cortex, the parietal eye field (PEF) is involved in computing the spatial location of the stimulus (65-67) and thus the vector of the required anti-saccade in spatial coordinates $(68,69)$. The basal ganglia may participate in inhibiting the pro-saccade and facilitate the anti-saccade (20, 70). Finally, the superior colliculus releases the fixation and makes the ultimate decision on the pro-saccade/anti-saccade execution. As expected, abnormalities of anti-saccades are extensively reported in parietal and frontal lobe dysfunctions. More specifically, human lesions and transcranial magnetic stimulation (TMS) interference in FEF have been associated with increased error rate, lesions of SEF, and pre-SMA with longer latency; while extensive lesions of frontal lobe have caused inability to perform the anti-saccadic task $(15,17,61,71,72)$. Moreover, PEF damage
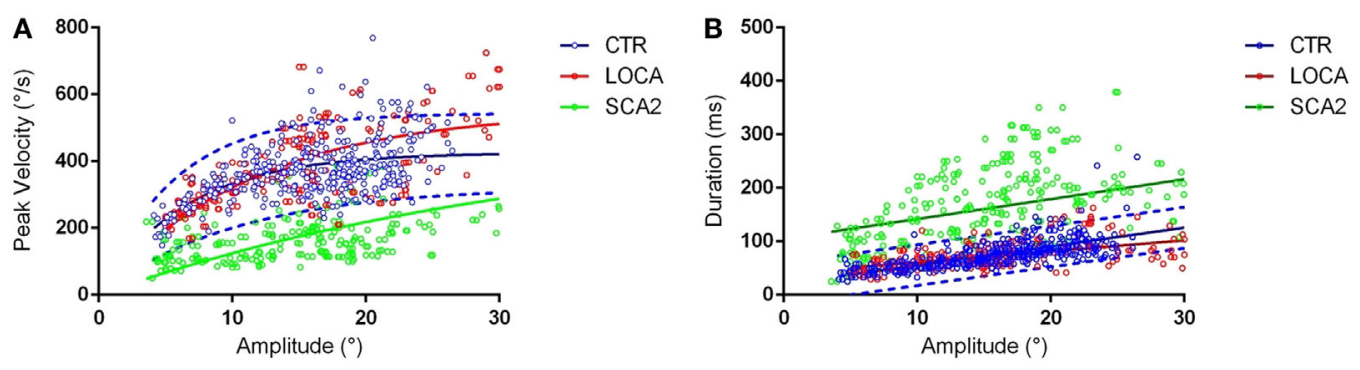

FIGURE 1 | (A) Main sequences amplitude-peak velocity. Group-based different patterns of the relationship between peak velocity and amplitude were suggested by exploration of the amplitude-peak velocity main sequence. The thick lines denote the main sequence equations fitted over the data. The blue dashed lines represent the 95\% prediction bound of the healthy controls. The scatter points of late onset cerebellar ataxia (LOCA) (red dots) and healthy controls (blue dots) overlapped almost everywhere, while the spinocerebellar ataxia type 2 (SCA2) data points (green dots) were outside the 95\% prediction bounds. (B) Main sequences amplitude-duration. The SCA2 patients (green dots) presented also a different pattern in the amplitude-duration main sequence. 

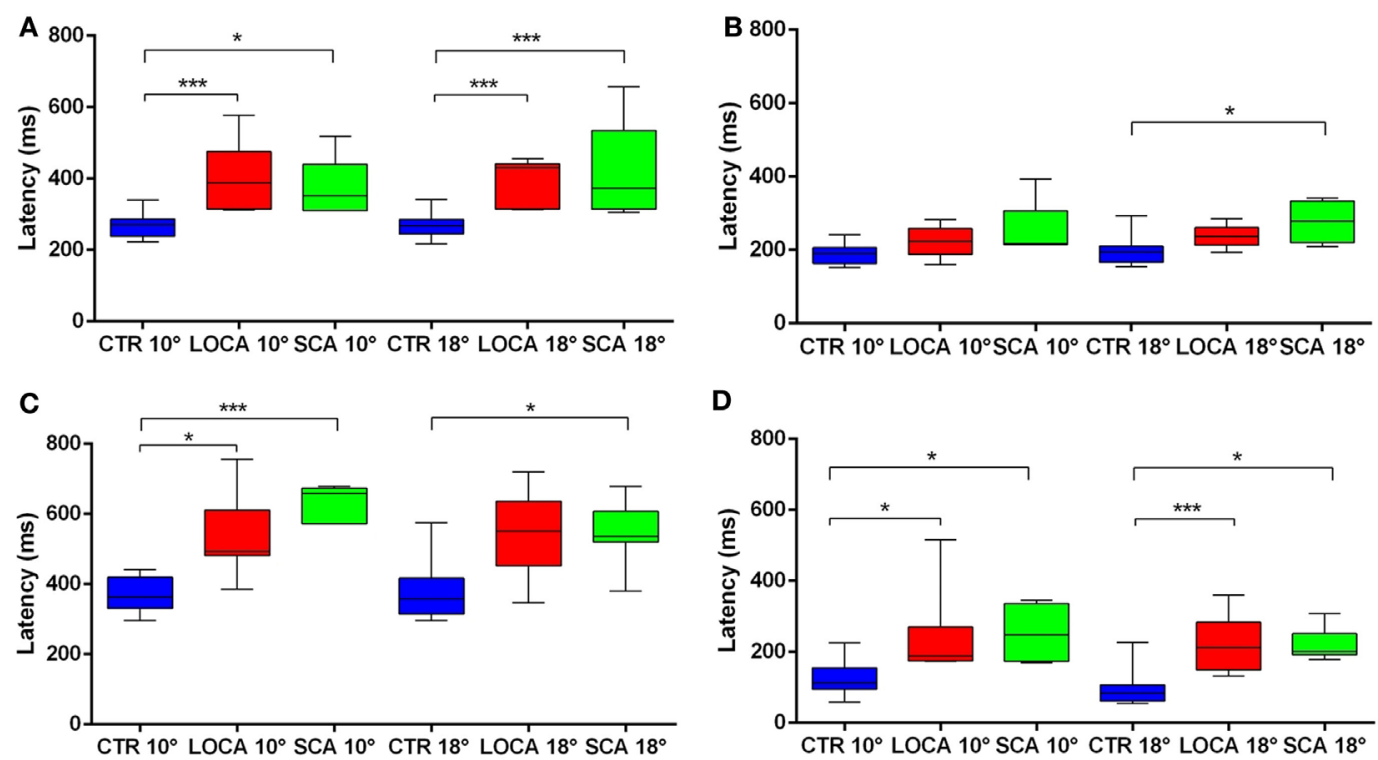

FIGURE 2 | (A-D) Box plots of latencies by group and eccentricity. A star code is used for representing the extent of the significance levels of the differences in the

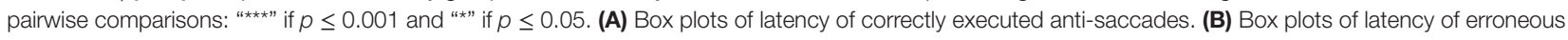
pro-saccades. (C) Box plots of latency of corrective anti-saccades. (D) Box plots of inter-saccadic latency.

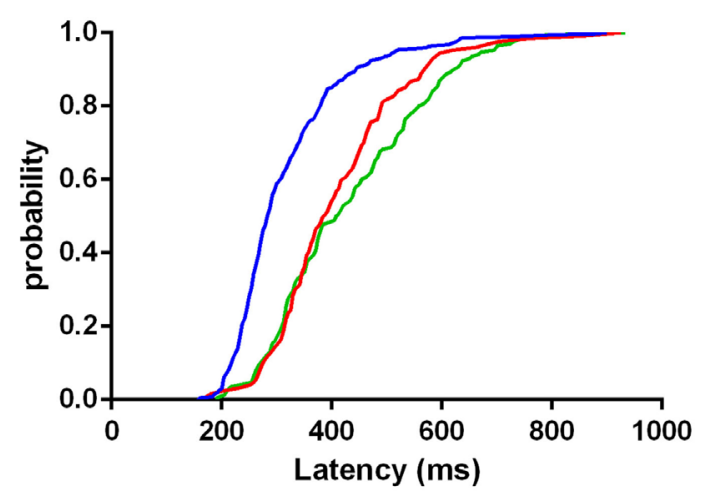

FIGURE 3 | Cumulative distribution functions of latencies in correctly executed anti-saccades of controls (blue curve), late onset cerebellar ataxia (red curve), and spinocerebellar ataxia type 2 (green curve). The plot indicates that the mass of the distribution is more concentrated on the shortest values of latency in controls than in patients.

and TMS application have been related to hypometric and less precise anti-saccades with longer latency $(51,73,74)$.

The cerebellum is also supposed to participate to this complex network by controlling voluntary eye movements through its connections with the frontal-parietal cortex $(75,76)$.

Indeed, anti-saccades increased error rate and prolonged latencies have been found in patients with Cerebrotendineous Xanthomatosis (24), Friedreich ataxia (25), Spinocerebellar Ataxia type 1 (26), SCA2 (27), focal cerebellar lesions (28), and degenerative cerebellar diseases (29-31). A significant correlation between high-error rates of anti-saccades and loss of gray matter volume in the posterolateral cerebellum has also been reported in neurodegenerative cerebellar diseases (77). This cerebellar region has been associated with complex motor and cognitive functions (78) and is also activated in concert with the pre-frontal cortex, via projections to the thalamus and SMA, during error processing and post error processing in stop signal and reversal tasks $(79,80)$.

However, most patients with cerebellar degenerative diseases, who have been enrolled in these studies, presented atrophy that was not confined to the cerebellum, but involved also cortical areas. Therefore, in these studies, while a role of the cerebellum can be strongly suspected, its contribution cannot be isolated.

To our knowledge, this is the first study in which two large groups of cerebellar patients with and without cortical involvement are compared in order to identify the role of the cerebellum in the anti-saccade execution. As anticipated above, according with previous studies, our results demonstrated that all cerebellar patients presented an increased rate of unwanted pro-saccades in the anti-saccadic task. Since no significant differences were found between SCA2 and LOCA, this observation suggests a direct control of the cerebellum on the frontal network in suppressing an unwanted reflexive movement when a competing voluntary movement is desired (37, 81-85). Thus, the cerebellum, which is reciprocally interconnected with frontal areas inhibiting reflexive movements (FEF and DLPFC), could play a central role in the decisional process of voluntary motor control.

Also, in agreement with previous studies, we found that cerebellar patients had increased and more variable anti-saccadic latency than the normal population $(14,22,25,27,77)$. Previously, it has been demonstrated that the latency of anti-saccades is usually more variable (trial by trial variability) and longer than that of visually guided saccades $(12,56)$. An influential model explaining 

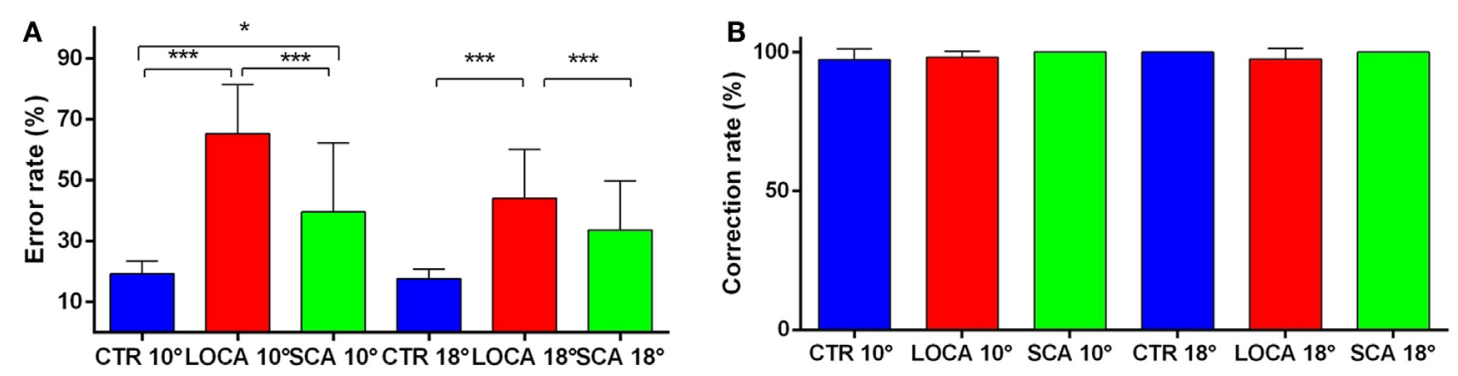

FIGURE 4 | (A) Mean and SE of the percentage of errors in the anti-saccade task in each group at 10 and 18 deg. A star code is used for representing the extent of the significance levels of the differences in the pairwise comparisons: "***" if $p \leq 0.001$ and "** if $p \leq 0.05$. (B) Mean and SE of the percentage of corrected anti-saccades with respect to the number of direction errors in each group at 10 and 18 deg.

the response time distribution in a simple decisional process such as pro-saccades vs anti-saccade, is the linear approach to threshold with ergodic rate model. This is a race-to-threshold model in which two different options compete for the final decision. According to rise-to-threshold models of anti-saccades $(61,86,87)$, we suggest that longer and variable latencies might reflect the unpredictable timing for the accumulation of information concerning the execution of a correct anti-saccade (88), or might result from noisy accumulation of information during the decisional process $(89,90)$. Since a cerebellar dysfunction alone seems to be sufficient to delay the latency of a wanted movement (an anti-saccade in this case), a specific role of the cerebellum in controlling the onset of a voluntary movement is likely. The cerebellum, thus, through its connections, might accelerate the information accumulation or reduce the noise in pre-frontal areas like the SEF and pre-SMA, and/or in the parietal areas as the PEF, which are involved in determining the anti-saccade onset.

As novel findings, we showed that cerebellar patients corrected their errors as frequently as normal, but the corrective anti-saccades occurred at a longer latency than normal.

In a previous study, we had already observed that, while the rate of anti-saccade errors increased with subcortical impairment (namely cerebellum and basal ganglia dysfunction), the rate of anti-saccade correction decreased only when also a cerebral cortical involvement and cognitive impairment was present (38). In this framework, the cerebellum would intervene in facilitating the suppression of unwanted reflexive movements (pro-saccades), but in case of errors, their correction would be mostly a cortical property. Also in this study, the impairment of the cerebellum was associated with increased directional error rate. Conversely, the well-maintained correction rate in our subjects might indicate a relative preservation of the cortical areas (as otherwise indicated by the absence of severe cognitive impairment in all our patients including the SCA2 patients). This would suggest an intrinsic monitoring of the failure/correction rate in the pre-frontal areas during goal-directed movements; on this rate of correction, subcortical areas such as the cerebellum would have little influence.

It is noteworthy that the corrective anti-saccades in the cerebellar patients occurred at longer intersaccadic intervals than normal. In normal subjects, the intersaccadic interval between erroneous pro-saccade and corrective anti-saccade was quite short for allowing a visual feedback and clearly shorter than the average latency of a correct anti-saccade. This indicates that the corrective anti-saccade was likely pre-planned and not generated after the visual feedback of the error. Also in cerebellar patients the error-correction intersaccadic interval was shorter than the correct anti-saccade latency, thus suggesting that the corrective anti-saccades were possibly pre-planned in their case as well.

Overall, we did not find significant differences between SCA2 and LOCA in anti-saccade behavior. This is perhaps surprising even considering a relative sparing of the cortical areas in our SCA2 patients, because we would have nevertheless expected a cumulative effect of their neurodegeration (cortex plus cerebellum) to result in a worse performance. The lack of significative difference might instead suggest that the cerebellum plays a crucial, bottleneck role in executive control of anti-saccades. Moreover, it might indicate that the cortical involvement (particularly the frontal lobe impairment) might be compensated by other areas, including those spared in the cerebellum. Supporting this hypothesis could be the finding of relatively spared dentate nuclei in the same SCA2 patients (91-93). This also suggests that the cerebellum has more than an ancillary supporting role in executive control, being able to compensate up to a certain measure a cortical failure.

In conclusion, our study sustains and reinforces the hypothesis that the cerebellum contributes actively to the control of executive functions. The cerebellum would participate in a functional network connecting the pre-frontal, frontal, and parietal cortex that facilitates the planning of voluntary movements while suppressing perturbing automatic responses. However, not all cortical computations might be under the same cerebellar control. Some cortical properties, such as the failure/correction rate, might be less easily influenced by the cerebellum.

\section{ETHICS STATEMENT}

All procedures performed in studies involving human participants were in accordance with the ethical standards of Local Ethic Committee: Comitato Etico Locale Azienda Ospedaliera Universitaria Senese and with the 1964 Helsinki declaration and its later amendments or comparable ethical standards. The protocol was approved by the local Ethical Committee (EVAlab protocol CEL no. 48/2010). Patients gave their written consent. 


\section{AUTHOR CONTRIBUTIONS}

EP performed and analyzed saccades, interpreted results, and wrote the paper; PP performed statistical analysis, interpreted results, and wrote tables and figures; FR collected clinical findings, performed, and analyzed saccades, interpreted results; PF implemented saccade analysis and interpreted results; VS wrote "Materials and Methods" paragraph and gave her contribution in saccade analysis and figure plotting; GT analyzed saccades and collected patient's data; MD and AF collected patients and defined the clinical and genetic diagnosis; AR evaluated patients, designed the study, interpreted, and wrote the paper.

\section{REFERENCES}

1. Hopp JJ, Fuchs AF. The characteristics and neuronal substrate of saccadic eye movement plasticity. Prog Neurobiol (2004) 72:27-53. doi:10.1016/j. pneurobio.2003.12.002

2. Catz N, Dicke PW, Thier P. Cerebellar complex spike firing is suitable to induce as well as to stabilize motor learning. Curr Biol (2005) 15:2179-89. doi:10.1016/j.cub.2005.11.037

3. Chen-Harris H, Joiner WM, Ethier V, Zee DS, Shadmehr R. Adaptive control of saccades via internal feedback. J Neurosci (2008) 28:2804-13. doi:10.1523/ JNEUROSCI.5300-07.2008

4. Kojima Y, Soetedjo R, Fuchs AF. Behavior of the oculomotor vermis for five different types of saccade. J Neurophysiol (2010) 104:3667-76. doi:10.1152/ jn.00558.2010

5. Allen G, Courchesne E. The cerebellum and non-motor function: clinical implications. Mol Psychiatry (1998) 3:207-10. doi:10.1038/sj.mp.4000395

6. Dum RP, Strick PL. An unfolded map of the cerebellar dentate nucleus and its projections to the cerebral cortex. J Neurophysiol (2003) 89:634-9. doi:10.1152/jn.00626.2002

7. Manto M, Lorivel T. Cognitive repercussions of hereditary cerebellar disorders. Cortex (2011) 47:81-100. doi:10.1016/j.cortex.2009.04.012

8. Hocking DR, Corben LA, Fielding J, Cremer PD, Millist L, White OB, et al. Saccade reprogramming in Friedreich ataxia reveals impairments in the cognitive control of saccadic eye movement. Brain Cogn (2014) 87:161-7. doi:10.1016/j.bandc.2014.03.018

9. Gao JH, Parsons LM, Bower JM, Xiong J, Li J, Fox PT. Cerebellum implicated in sensory acquisition and discrimination rather than motor control. Science (1996) 272:545-7. doi:10.1126/science.272.5261.545

10. Blakemore SJ, Frith CD, Wolpert DM. Spatio-temporal prediction modulates the perception of self-produced stimuli. J Cogn Neurosci (1999) 11:551-9. doi:10.1162/089892999563607

11. Blakemore SJ, Wolpert DM, Frith CD. The cerebellum contributes to somatosensory cortical activity during self-produced tactile stimulation. Neuroimage (1999) 10:448-59. doi:10.1006/nimg.1999.0478

12. Peterburs J, Desmond JE. The role of the human cerebellum in performance monitoring. Curr Opin Neurobiol (2016) 40:38-44. doi:10.1016/j. conb.2016.06.011

13. Buckner RL. The cerebellum and cognitive function: 25 years of insight from anatomy and neuroimaging. Neuron (2013) 80:807-15. doi:10.1016/j. neuron.2013.10.044

14. Hallett PE. Primary and secondary saccades to goals defined by instructions. Vision Res (1978) 18:1279-96. doi:10.1016/0042-6989(78)90218-3

15. Terao Y, Fukuda H, Ugawa Y, Hikosaka O, Hanajima R, Furubayashi T, et al. Visualization of the information flow through human oculomotor cortical regions by transcranial magnetic stimulation. J Neurophysiol (1998) 80:936-46. doi:10.1152/jn.1998.80.2.936

16. Middleton FA, Strick PL. Cerebellar projections to the prefrontal cortex of the primate. JNeurosci (2001) 21:700-12. doi:10.1523/JNEUROSCI.2102-00700.2001

17. Curtis CE, D’Esposito M. Success and failure suppressing reflexive behavior. J Cogn Neurosci (2003) 15:409-18. doi:10.1162/089892903321593126

\section{ACKNOWLEDGMENTS}

We kindly thank the patients and their families for their participation in the study. We also thank Dr. Alfonso Cerase and Dr. Lucia Monti, who performed neuroimaging examinations of the patients.

\section{FUNDING}

Study in part funded by EC FP7-PEOPLE-IRSES-CERVISO 269263; RT_BRAIHURO_POR CReO 2007-2013_1.5a_1.6. and the Intramural Research Program of NEI.

18. Ford KA, Goltz HC, Brown MR, Everling S. Neural processes associated with antisaccade task performance investigated with event-related FMRI. J Neurophysiol (2005) 94:429-40. doi:10.1152/jn.00471.2004

19. McDowell JE, Kissler JM, Berg P, Dyckman KA, Gao Y, Rockstroh B, et al. Electroencephalography/magnetoencephalography study of cortical activities preceding prosaccades and antisaccades. Neuroreport (2005) 16:663-8. doi:10.1097/00001756-200505120-00002

20. Ettinger U, Ffytche DH, Kumari V, Kathmann N, Reuter B, Zelaya F, et al. Decomposing the neural correlates of antisaccade eye movements using event-related FMRI. Cereb Cortex (2008) 18:1148-59. doi:10.1093/cercor/ bhm 147

21. de Weijer AD, Mandl RC, Sommer IE, Vink M, Kahn RS, Neggers SF. Human fronto-tectal and fronto-striatal-tectal pathways activate differently during anti-saccades. Front Hum Neurosci (2010) 4:41. doi:10.3389/fnhum.2010. 00041

22. Prevosto V, Graf W, Ugolini G. Cerebellar inputs to intraparietal cortex areas LIP and MIP: functional frameworks for adaptive control of eye movements, reaching, and arm/eye/head movement coordination. Cereb Cortex (2010) 20:214-28. doi:10.1093/cercor/bhp091

23. Ramnani N. Frontal lobe and posterior parietal contributions to the cortico-cerebellar system. Cerebellum (2012) 11:366-83. doi:10.1007/s12311011-0272-3

24. Rosini F, Pretegiani E, Mignarri A, Optican LM, Serchi V, De Stefano N, et al. The role of dentate nuclei in human oculomotor control: insights from cerebrotendinous xanthomatosis. J Physiol (2017) 595:3607-20. doi:10.1113/ JP273670

25. Fielding J, Corben L, Cremer P, Millist L, White O, Delatycki M. Disruption to higher order processes in Friedreich ataxia. Neuropsychologia (2010) 48:235-42. doi:10.1016/j.neuropsychologia.2009.09.009

26. Rivaud-Pechoux S, Dürr A, Gaymard B, Cancel G, Ploner CJ, Agid Y, et al. Eye movement abnormalities correlate with genotype in autosomal dominant cerebellar ataxia type I. Ann Neurol (1998) 43:297-302. doi:10.1002/ana. 410430306

27. Rodríguez-Labrada R, Velázquez-Pérez L, Aguilera-Rodríguez R, SeifriedOberschmidt C, Peña-Acosta A, Canales-Ochoa N, et al. Executive deficit in spinocerebellar ataxia type 2 is related to expanded CAG repeats: evidence from antisaccadic eye movements. Brain Cogn (2014) 91:28-34. doi:10.1016/j. bandc.2014.07.007

28. Brunamonti E, Chiricozzi FR, Clausi S, Olivito G, Giusti MA, Molinari M, et al. Cerebellar damage impairs executive control and monitoring of movement generation. PLoS One (2014) 9:e85997. doi:10.1371/journal.pone.0085997

29. King SA, Schneider RM, Serra A, Leigh RJ. Critical role of cerebellar fastigial nucleus in programming sequences of saccades. Ann N Y Acad Sci (2011) 1233:155-61. doi:10.1111/j.1749-6632.2011.06119.x

30. Filippopulos F, Eggert T, Straube A. Deficits of cortical oculomotor mechanisms in cerebellar atrophy patients. Exp Brain Res (2013) 224:541-50. doi:10.1007/s00221-012-3332-0

31. Jamadar SD, Johnson BP, Clough M, Egan GF, Fielding J. Behavioral and neural plasticity of ocular motor control: changes in performance and fMRI activity following antisaccade training. Front Hum Neurosci (2015) 9:653. doi:10.3389/fnhum.2015.00653 
32. Robinson DA. Oculomotor control signals. In: Lennerstrand F, Bach-y-Rita P, editors. Basic Mechanisms of Ocular Motility and Their Clinical Implications. Oxford: Pergamon Press (1975). p. 337-74.

33. Dieterich M, Bucher SF, Seelos KC, Brandt T. Cerebellar activation during optokinetic stimulation and saccades. Neurology (2000) 54:148-55. doi:10.1212/ WNL.54.1.148

34. Robinson FR, Fuchs AF. The role of the cerebellum in voluntary eye movements. Annu Rev Neurosci (2001) 24:981-1004. doi:10.1146/annurev.neuro. 24.1.981

35. Nitschke MF, Binkofski F, Buccino G, Posse S, Erdmann C, Kömpf D, et al. Activation of cerebellar hemispheres in spatial memorization of saccadic eye movements: an fMRI study. Hum Brain Mapp (2004) 22:155-64. doi:10.1002/ hbm. 20025

36. Nitschke MF, Arp T, Stavrou G, Erdmann C, Heide W. The cerebellum in the cerebro-cerebellar network for the control of eye and hand movements-an fMRI study. Prog Brain Res (2005) 148:151-64. doi:10.1016/ S0079-6123(04)48013-3

37. Strick PL, Dum RP, Fiez JA. Cerebellum and nonmotor function. Annu Rev Neurosci (2009) 32:413-34. doi:10.1146/annurev.neuro.31.060407.125606

38. Pretegiani E, Rosini F, Federico A, Optican LM, Rufa A. Eye movements in genetic parkinsonisms affecting the $\alpha$-synuclein, PARK9, and manganese network. Clin Neurophysiol (2017) 128:2450-3. doi:10.1016/j.clinph.2017. 09.107

39. Kerber KA, Jen JC, Perlman S, Baloh RW. Late-onset pure cerebellar ataxia: differentiating those with and without identifiable mutations. J Neurol Sci (2005) 238:41-5. doi:10.1016/j.jns.2005.06.006

40. Magaña JJ, Velázquez-Pérez L, Cisneros B. Spinocerebellar ataxia type 2: clinical presentation, molecular mechanisms, and therapeutic perspectives. Mol Neurobiol (2013) 47:90-104. doi:10.1007/s12035-012-8348-8

41. Lastres-Becker I, Rüb U, Auburger G. Spinocerebellar ataxia 2 (SCA2). Cerebellum (2008) 7:115-24. doi:10.1007/s12311-008-0019-y

42. Rüb U, Schöls L, Paulson H, Auburger G, Kermer P, Jen JC, et al. Clinical features, neurogenetics and neuropathology of the polyglutamine spinocerebellar ataxias type 1, 2, 3, 6 and 7. Prog Neurobiol (2013) 104:38-66. doi:10.1016/j. pneurobio.2013.01.001

43. Trouillas P, Takayanagi T, Hallett M, Currier RD, Subramony SH, Welles K, et al. International cooperative ataxia rating scale for pharmacological assessment of the cerebellar syndrome. The ataxia neuropharmacology committee of the world federation of neurology. J Neurol Sci (1997) 145:205-11. doi:10.1016/S0022-510X(96)00231-6

44. Leigh RJ, Zee DS. The Neurology of Eye Movements. 5th ed. New York: Oxford University Press (2015).

45. Inchingolo P, Spanio M. On the identification and analysis of saccadic eye movements - a quantitative study of the processing procedures. IEEE Trans Biomed Eng (1985) 32:683-95. doi:10.1109/TBME.1985.325586

46. Pareyson D, Gellera C, Castellotti B, Antonelli A, Riggio MC, Mazzucchelli F, et al. Clinical and molecular studies of 73 Italian families with autosomal dominant cerebellar ataxia type I: SCA1 and SCA2 are the most common genotypes. J Neurol (1999) 246:389-93. doi:10.1007/s004150050369

47. Hübner J, Sprenger A, Klein C, Hagenah J, Rambold H, Zühlke C, et al. Eye movement abnormalities in spinocerebellar ataxia type 17 (SCA17). Neurology (2007) 69:1160-8. doi:10.1212/01.wnl.0000276958.91986.89

48. Shelton AL, Cornish K, Kraan C, Georgiou-Karistianis N, Metcalfe SA, Bradshaw JL, et al. Exploring inhibitory deficits in female premutation carriers of fragile X syndrome: through eye movements. Brain Cogn (2014) 85:201-8. doi:10.1016/j.bandc.2013.12.006

49. Federighi P, Cevenini G, Dotti MT, Rosini F, Pretegiani E, Federico A, et al. Differences in saccade dynamics between spinocerebellar ataxia 2 and late-onset cerebellar ataxias. Brain (2011) 134:879-91. doi:10.1093/brain/ awr009

50. Krappmann P, Everling S, Flohr H. Accuracy of visually and memory-guided antisaccades in man. Vision Res (1998) 38:2979-85. doi:10.1016/ S0042-6989(98)00101-1

51. Gaymard B, Lynch J, Ploner CJ, Condy C, Rivaud-Péchoux S. The parieto-collicular pathway: anatomical location and contribution to saccade generation. Eur J Neurosci (2003) 17:1518-26. doi:10.1046/j.1460-9568.2003.02570.x

52. Pierrot-Deseilligny C, Müri RM, Ploner CJ, Gaymard B, Demeret S, RivaudPechoux S. Decisional role of the dorsolateral prefrontal cortex in ocular motor behaviour. Brain (2003) 126:1460-73. doi:10.1093/brain/awg148
53. Pierrot-Deseilligny C, Milea D, Müri RM. Eye movement control by the cerebral cortex. Curr Opin Neurol (2004) 17:17-25. doi:10.1097/00019052200402000-00005

54. Bruce CJ, Goldberg ME. Primate frontal eye fields. I. Single neurons discharging before saccades. J Neurophysiol (1985) 53:603-35. doi:10.1152/ jn.1985.53.3.603

55. Everling S, Johnston K. Control of the superior colliculus by the lateral prefrontal cortex. Philos Trans R Soc Lond B Biol Sci (2013) 368:20130068. doi:10.1098/rstb.2013.0068

56. Everling S, Munoz DP. Neuronal correlates for preparatory set associated with prosaccades and anti-saccades in the primate frontal eye field. J Neurosci (2000) 20:387-400. doi:10.1523/JNEUROSCI.20-01-00387.2000

57. Guitton D, Buchtel HA, Douglas RM. Frontal lobe lesions in man cause difficulties in suppressing reflexive glances and in generating goal-directed saccades. Exp Brain Res (1985) 58:455-72. doi:10.1007/BF00235863

58. Connolly JD, Goodale MA, Menon RS, Munoz DP. Human fMRI evidence for the neural correlates of preparatory set. Nat Neurosci (2002) 5:1345-52. doi:10.1038/nn969

59. Jamadar SD, Fielding J, Egan GF. Quantitative meta-analysis of fMRI and PET studies reveals consistent activation in fronto-striatal-parietal regions and cerebellum during antisaccades and prosaccades. Front Psychol (2013) 4:749. doi:10.3389/fpsyg.2013.00749

60. Schlag-Rey M, Amador N, Sanchez H, Schlag J. Antisaccade performance predicted by neuronal activity in the supplementary eye field. Nature (1997) 390:398-401. doi:10.1038/37114

61. Stuphorn V, Schall JD. Neuronal control and monitoring of initiation of movements. Muscle Nerve (2002) 26:326-39. doi:10.1002/mus.10158

62. Amador N, Schlag-Rey M, Schlag J. Primate antisaccade. II. Supplementary eye field neuronal activity predicts correct performance. J Neurophysiol (2004) 91:1672-89. doi:10.1152/jn.00138.2003

63. Carpenter RH. Contrast, probability, and saccadic latency; evidence for independence of detection and decision. Curr Biol (2004) 14:1576-80. doi:10.1016/j.cub.2004.08.058

64. Botvinick MM, Cohen JD, Carter CS. Conflict monitoring and anterior cingulate cortex: an update. Trends Cogn Sci (2004) 8:539-46. doi:10.1016/j. tics.2004.10.003

65. Duhamel JR, Goldberg ME, Fitzgibbon EJ, Sirigu A, Grafman J. Saccadic dysmetria in a patient with a right frontoparietal lesion. The importance of corollary discharge for accurate spatial behaviour. Brain (1992) 115:1387-402. doi:10.1093/brain/115.5.1387

66. Colby CL, Goldberg ME. Space and attention in parietal cortex. Annu Rev Neurosci (1999) 22:319-49. doi:10.1146/annurev.neuro.22.1.319

67. Gottlieb J, Goldberg ME. Activity of neurons in the lateral intraparietal area of the monkey during an antisaccade task. Nat Neurosci (1999) 2:906-12. doi:10.1038/13209

68. Zhang M, Barash S. Neuronal switching of sensorimotor transformations for antisaccades. Nature (2000) 408:971-5. doi:10.1038/35050097

69. Zhang M, Barash S. Persistent LIP activity in memory antisaccades: working memory for a sensorimotor transformation. J Neurophysiol (2004) 91:1424-41. doi:10.1152/jn.00504.2003

70. Kim HF, Hikosaka O. Parallel basal ganglia circuits for voluntary and automatic behaviour to reach rewards. Brain (2015) 138:1776-800. doi:10.1093/ brain/awv134

71. Schlag-Rey M, Schlag J, Dassonville P. How the frontal eye field can impose a saccade goal on superior colliculus neurons. J Neurophysiol (1992) 67:1003-5. doi:10.1152/jn.1992.67.4.1003

72. Boxer AL, Garbutt S, Rankin KP, Hellmuth J, Neuhaus J, Miller BL, et al. Medial versus lateral frontal lobe contributions to voluntary saccade control as revealed by the study of patients with frontal lobe degeneration. J Neurosci (2006) 26:6354-63. doi:10.1523/JNEUROSCI.0549-06.2006

73. Gottlieb J, Kusunoki M, Goldberg ME. Simultaneous representation of saccade targets and visual onsets in monkey lateral intraparietal area. Cereb Cortex (2005) 15:1198-206. doi:10.1093/cercor/bhi002

74. Andersen RA, Brotchie PR, Mazzoni P. Evidence for the lateral intraparietal area as the parietal eye field. Curr Opin Neurobiol (1992) 2:840-6 doi:10.1016/0959-4388(92)90143-9

75. Middleton FA, Strick PL. Anatomical evidence for cerebellar and basal ganglia involvement in higher cognitive function. Science (1994) 266:458-61. doi:10.1126/science.7939688 
76. Stoodley CJ. The cerebellum and cognition: evidence from functional imaging studies. Cerebellum (2012) 11:352-65. doi:10.1007/s12311-0110260-7

77. Peterburs J, Thürling M, Rustemeier M, Göricke S, Suchan B, Timmann D, et al. A cerebellar role in performance monitoring-evidence from EEG and voxel-based morphometry in patients with cerebellar degenerative disease. Neuropsychologia (2015) 68:139-47. doi:10.1016/j.neuropsychologia. 2015.01.017

78. Stoodley CJ, Schmahmann JD. Functional topography in the human cerebellum: a meta-analysis of neuroimaging studies. Neuroimage (2009) 44:489-501. doi:10.1016/j.neuroimage.2008.08.039

79. Ide JS, Li CS. A cerebellar thalamic cortical circuit for error-related cognitive control. Neuroimage (2011) 54:455-64. doi:10.1016/j.neuroimage.2010. 07.042

80. Von der Gablentz J, Tempelmann C, Münte TF, Heldmann M. Performance monitoring and behavioral adaptation during task switching: an fMRI study. Neuroscience (2015) 285:227-35. doi:10.1016/j.neuroscience.2014. 11.024

81. Okuda B. Cerebello-thalamo-cerebral projection from the dentate nucleus onto the frontal eye field in the cat. Acta Physiol Scand (1994) 151:1-6. doi:10.1111/j.1748-1716.1994.tb09715.x

82. Luna B, Thulborn KR, Munoz DP, Merriam EP, Garver KE, Minshew NJ, et al. Maturation of widely distributed brain function subserves cognitive development. Neuroimage (2001) 13:786-93. doi:10.1006/nimg. 2000.0743

83. Nagao S. Pontine nuclei-mediated cerebello-cerebral interactions and its functional role. Cerebellum (2004) 3:11-5. doi:10.1080/14734220310012181

84. Prevosto V, Sommer MA. Cognitive control of movement via the cerebellarrecipient thalamus. Front Syst Neurosci (2013) 7:56. doi:10.3389/fnsys. 2013.00056

85. Hashimoto M, Takahara D, Hirata Y, Inoue K, Miyachi S, Nambu A, et al. Motor and non-motor projections from the cerebellum to rostrocaudally distinct sectors of the dorsal premotor cortex in macaques. Eur J Neurosci (2010) 31:1402-13. doi:10.1111/j.1460-9568.2010.07151.x

86. Kristjánsson A, Chen Y, Nakayama K. Less attention is more in the preparation of antisaccades, but not prosaccades. Nat Neurosci (2001) 4:1037-42. doi:10.1038/nn723
87. Munoz DP, Everling S. Look away: the anti-saccade task and the voluntary control of eye movement. Nat Rev Neurosci (2004) 5:218-28. doi:10.1038/ nrn1345

88. Noorani I, Carpenter RH. Antisaccades as decisions: LATER model predicts latency distributions and error responses. Eur J Neurosci (2013) 37:330-8. doi:10.1111/ejn.12025

89. Cutsuridis V, Smyrnis N, Evdokimidis I, Perantonis S. A neural model of decision-making by the superior colicullus in an antisaccade task. Neural Netw (2007) 20:690-704. doi:10.1016/j.neunet.2007.01.004

90. Cutsuridis V, Kumari V, Ettinger U. Antisaccade performance in schizophrenia: a neural model of decision making in the superior colliculus. Front Neurosci (2014) 8:13. doi:10.3389/fnins.2014.00013

91. Mascalchi M, Diciotti S, Giannelli M, Ginestroni A, Soricelli A, Nicolai E, et al. Progression of brain atrophy in spinocerebellar ataxia type 2: a longitudinal tensor-based morphometry study. PLoS One (2014) 9:e89410. doi:10.1371/ journal.pone.0089410

92. Della Nave R, Ginestroni A, Tessa C, Salvatore E, De Grandis D, Plasmati R, et al. Brain white matter damage in SCA1 and SCA2. An in vivo study using voxel-based morphometry, histogram analysis of mean diffusivity and tract-based spatial statistics. Neuroimage (2008) 43:10-9. doi:10.1016/j. neuroimage.2008.06.036

93. Peterburs J, Gajda K, Koch B, Schwarz M, Hoffmann KP, Daum I, et al. Cerebellar lesions alter performance monitoring on the antisaccade task-an event-related potentials study. Neuropsychologia (2012) 50(3):379-89. doi:10.1016/ j.neuropsychologia.2011.12.009

Conflict of Interest Statement: The authors declare that the research was conducted in the absence of any commercial or financial relationships that could be construed as a potential conflict of interest.

Copyright (c) 2018 Pretegiani, Piu, Rosini, Federighi, Serchi, Tumminelli, Dotti, Federico and Rufa. This is an open-access article distributed under the terms of the Creative Commons Attribution License (CC BY). The use, distribution or reproduction in other forums is permitted, provided the original author (s) and the copyright owner are credited and that the original publication in this journal is cited, in accordance with accepted academic practice. No use, distribution or reproduction is permitted which does not comply with these terms. 